\title{
Hereditary symbionts and mitochondria: distribution in insect populations and quasi-linkage of genetic markers
}

\author{
Ilya Zakharov and Elena Shaikevich \\ Vavilov Institute of General Genetics, Russian Academy of Sciences, \\ ul. Gubkina, 3, Moscow, 117971, Russian Federation \\ Address correspondence and requests for materials to llya Zakharov, iaz34@mail.ru
}

\begin{abstract}
Maternal transmission ensures the joint transmission and simultaneous presence in populations of individuals with certain variants of the bacterial symbiont and host mitochondrial DNA. Such "quasi-linkage" of cytoplasmic genomes among insects and other arthropods is widespread. The symbiont acts as a "driver" of mitochondria and the obvious biological consequence is the spread of the "linked" mitochondrial haplotype in the population, which itself does not have increased selective value to the organism. Examples of such indirect selective mitochondrial sweep in insects are discussed, as well as biological consequences of this phenomenon and mechanisms of increasing the frequency of symbiont-infected individuals in the population.
\end{abstract}

Keywords: symbiogenome, cytoplasmic genomes, insects, hereditary symbiont, mitochondrial haplotype, co-transmission

Citation: Zakharov, I. and Shaikevich, E. 2021. Hereditary symbionts and mitochondria: distribution in insect populations and quasi-linkage of genetic markers. Bio. Comm. 66(1): 6-16. https:// doi.org/10.21638/spbu03.2021.102

Authors' information: Ilya Zakharov, RAS Corresponding Member, Dr. of Sci. in Biology, Professor, orcid.org/0000-00024759-5940; Elena Shaikevich, PhD, Senior Researcher, orcid.org/0000-0002-6504-5547

Manuscript Editor: Anton Nizhnikov, Department of Genetics and Biotechnology, Faculty of Biology, Saint Petersburg State University, Saint Petersburg, Russia

Received: September 1, 2020;

Revised: December 1, 2020;

Accepted: December 16, 2020.

Copyright: ( 2021 Zakharov and Shaikevich. This is an open-access article distributed under the terms of the License Agreement with Saint Petersburg State University, which permits to the authors unrestricted distribution, and self-archiving free of charge.

Funding: The work of Zakharov I.A was supported by the Russian Science Foundation, Project 16-16-00079-P. The work of Shaikevich E.V. was supported by the Russian Foundation for Basic Research, Project 19-04-00739.

Competing interests: The authors have declared that no competing interests exist.

\section{Introduction}

Hereditary symbionts (Lederberg, 1952; Preer, 1971) are symbiotic microorganisms, usually bacteria that live inside cells and persist during their divisions. In most cases, these microorganisms are not able to exist outside the host cells. They are classified into obligate microorganisms, necessary for maintaining the life of the macroorganism, and facultative ones, which infect only a part of individuals in the host population. Intracellular symbionts are especially common among insects, and they are also known in other arthropods.

Facultative hereditary symbionts in some cases have a well-marked effect on the host phenotype; in other cases, their effects are little or apparently absent. The presence of a symbiont in cells is usually detected by taking into account certain DNA markers characteristic of the genome of this symbiotic bacterium.

The interaction of the genomes of the symbiont and host at the genetic and functional levels allows them to be considered as a composite genome, as a single genetic system, aptly named "symbiogenome" (Tikhonovich and Provorov, 2010; Provorov and Tikhonovich, 2014).

Hereditary symbionts are not only preserved in daughter cells when an infected mother cell divides, they are also transmitted to offspring during reproduction. The study of how they are inherited in this case has shown that transmission almost always occurs in the female line, that is, from the mother to the offspring. It is also known that the mitochondrial genome is usually transmitted through the female line as well. Thus, there is a joint transfer of two parts of the symbiogenome - the symbiont genome and mitochondrial DNA (mtDNA), which can be traced by taking into account the corresponding markers of these two genomes. The joint transmission also ensures the joint presence in populations of individuals with certain variants of the symbiont and mtDNA. 
In the literature, for the phenomenon of joint transmission and simultaneous presence in populations of the corresponding variants of the genomes of the symbiont and mitochondria, a term borrowed from classical population genetics is used, "linkage disequilibrium". The authors suppose that the term "quasi-linkage", which will be used later in this article, will be more appropriate.

This paper presents the results of studies conducted on two subjects - mosquitoes (Culex and Aedes) and beetles of the Adalia genus. In conclusion, the data obtained by other researchers when studying various objects are briefly reviewed.

\section{Mosquitoes}

\section{Coevolution of Wolbachia and host mtDNA in the Culex pipiens mosquito complex}

The Culex pipiens complex currently includes five contemporarily recognized taxa: $C x$. quinquefasciatus, $C x$. pipiens, Cx. pipiens pallens, Cx. australicus and Cx. globocoxitus. Cx. australicus and Cx. globocoxitus are Australian endemic taxa. $C x$. p. pallens is endemic in Japan. $C x$. quinguefasciatus is widely distributed in tropical and subtropical regions, and $C x$. pipiens is ubiquitous in temperate regions. The latter species includes mosquitoes of the pipiens and molestus forms with pronounced behavioral and physiological characteristics (Fedorova and Shaikevich, 2007). Mosquitoes of the form molestus are autogenous (first oviposition without a prior blood meal), stenogamous (able to mate in a narrow space and do not form a swarm), and anthropophilous (prefer biting mammals, including humans), and they do not have winter diapause. On the contrary, mosquitoes of the form pipiens are anautogenous (require a blood meal for egg development), eurygamous (need a lot of space for mating, swarming), diapause-forming and ornithophilic (they prefer biting birds). In temperate regions, $C x$. pipiens $\mathrm{f}$. molestus mostly reproduce in the basements of urban apartment buildings (Vinogradova et al., 2007), but they breed aboveground in the Mediterranean region (Gomes et al., 2009). Mosquitoes of the tropical subspecies $C x$. quinguefasciatus are anautogenous, stenogamous, anthropophilous, and have no diapause.

$C x$. torrentium is a cryptic species, closely related to $C x$. pipiens (Fedorova and Shaikevich, 2007). Vinogradova (see Shaikevich et al., 2006) has successfully crossed mosquitoes in the laboratory; hybrids had nuclear DNA from both $C x$. pipiens and Cx. torrentium. In nature, hybrids between two species were not found (Fedorova and Shaikevich, 2007). Cx. torrentium and $C x$. pipiens $\mathrm{f}$. pipiens have similar biological features: both are non-autogenous, diapausing, and eurygamous. $C x$. torrentium is widely distributed in the Palearctic, and both species often inhabit the same water bodies. Only minor morphological differences exist in their male genitalia. $C x$. torrentium and $C x$. pipiens originated from a common ancestor based on the phylogenetic analyses (Shaikevich and Zakharov, 2010; Dumas et al., 2016).

Mosquitoes of the Culex pipiens complex were characterized by the coinheritance of mtDNA and symbiotic bacteria Wolbachia (Rasgon et al., 2006; Dumas et al., 2013; Shaikevich and Zakharov, 2014; Shaikevich et al., 2016).

\section{MITOCHONDRIAL DNA DIVERSITY IN WOLBACHIA-INFECTED AND UNINFECTED CULEX MOSQUITOES}

The sequence diversity of the mitochondrial nicotinamide adenine dinucleotide (NADH) dehydrogenase subunit 4 (ND4) gene was investigated in Wolbachia-infected (South Africa (SA), California and Thailand) and uninfected (SA) Culex pipiens s.l. populations. In total, 12 mtDNA haplotypes (A-L) were identified. In infected populations, $99 \%$ of individuals had a single haplotype $\mathrm{K}$. In the uninfected SA population, 11 haplotypes were present, including K (Rasgon et al., 2006). Recently, three new mtDNA haplogroups were found in Wolbachia-uninfected specimens, and a different one in Wolbachiainfected mosquitoes within the Culex pipiens mosquito complex (Dumas et al., 2016). Concatenated sequences of the NADH dehydrogenase subunit 2 (ND2), subunit 4 (ND4) and cytochrome oxidase I (COI) genes of $\mathrm{mtD}$ NA were analyzed (Dumas et al., 2016).

The polymorphism of the full-size DNA sequence of the COI gene (1548 bp) was studied in Wolbachiainfected Culex pipiens f. pipiens and f. molestus and Wolbachia-uninfected Cx. torrentium from 16 locations in Russia and in 3 laboratory strains of subtropical Wolbachia-infected Cx. quinguefasciatus and Cx. pallens (Shaikevich and Zakharov, 2010). In 16 populations of the studied mosquito species, 15 haplotypes were found $(\mathrm{A}-\mathrm{O}) ; 10$ of these were detected in the Wolbachia-uninfected population of $C x$. torrentium. Three haplotypes were detected for $C x$. pipiens f. pipiens $(\mathrm{A}, \mathrm{B}, \mathrm{C})$; these haplotypes were not population-specific (Fig. 1). CX. p. pallens, which is considered a variety of $C x$. pipiens (Harbach, 2012), have a COI sequence identical with the main haplotype of Cx. f. pipiens (A). Cx. quinquefasciatus have haplotypes $\mathrm{E}$ and $\mathrm{E} 1$. Monomorphic haplotypes were found in Cx. pipiens f. molestus (D) (Fig. 1). The number of haplotypes in $C x$. pipiens was low, and the same haplotypes were found in distant populations.

Based on the DNA variability of the COI gene of both forms of Cx. pipiens and Cx. Quinquefasciatus, we developed restriction fragment length polymorphism (PCR-RFLP) assays (Shaikevich, 2007; Shaikevich, 2009). HaeIII digestion of the COI PCR products allowed the discrimination of the $\mathrm{D}$ haplotype from $\mathrm{A}, \mathrm{B}$, 


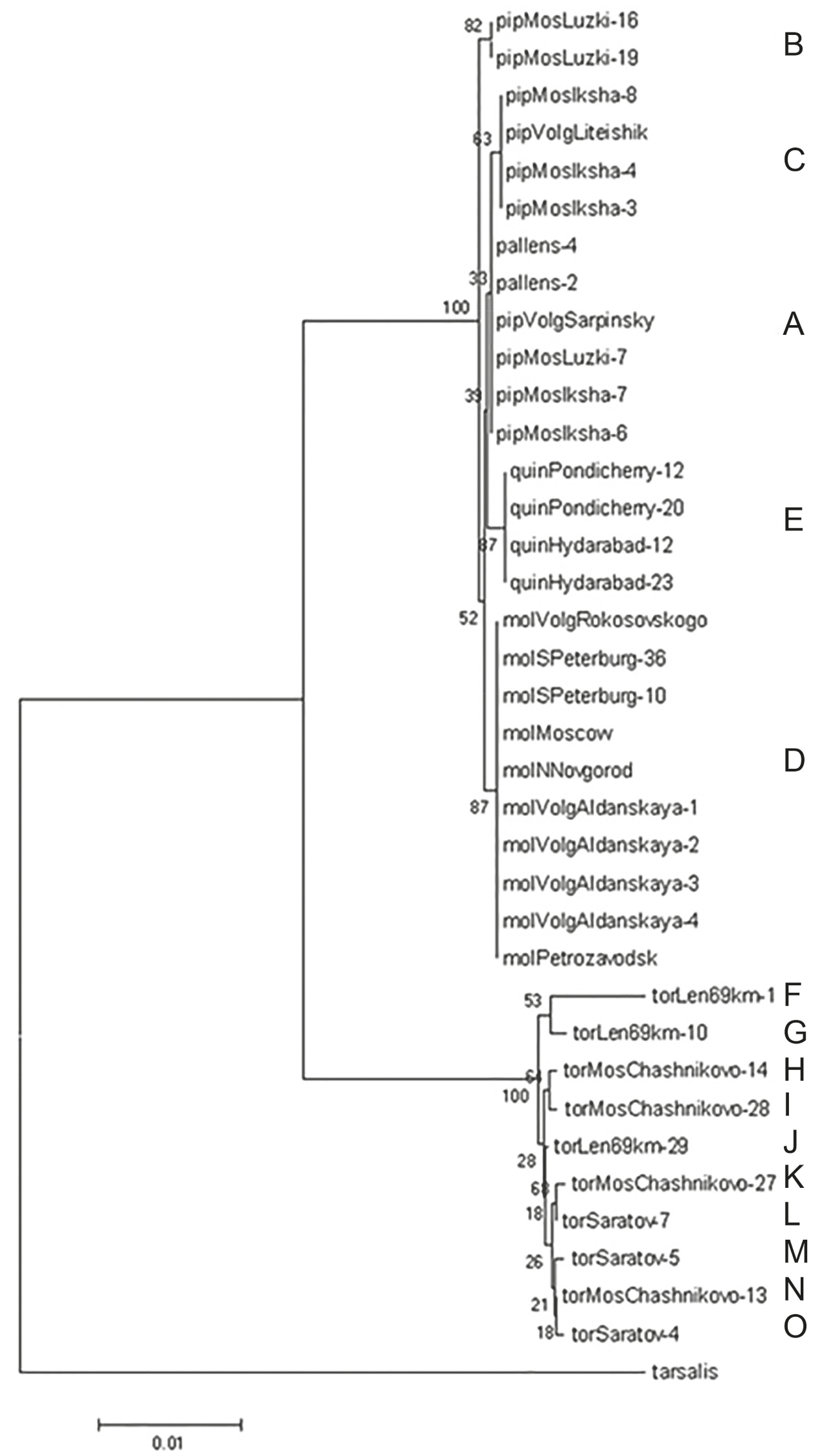

Fig. 1. The similarity dendrogram obtained for nucleotide sequences of $1548 \mathrm{bp}$ of the gene $C O /$ from mosquitoes of the $C x$. pipiens complex (Shaikevich and Zakharov, 2010): quin $-C x$. quinquefasciatus, pallens $-C x$. pallens, pip $-C x$. pipiens f. pipiens, mol $-C X$. pipiens f. molestus) and Cx. torrentium (tor). The haplotypes are marked by letters on the right. Mos - Moscow, Volg - Volgograd, Len69 - Leningrad obl., Pondichery and Hydarabad - origin of CX. quinquefasciatus (India). C. tarsalis (AF425847) was used as an external reference. 
$\mathrm{C}$ and $\mathrm{E}$ haplotypes. Alu I allows the discrimination of $\mathrm{E}$ and $\mathrm{E} 1$ haplotypes from others. Haplotypes A, B and C cannot be differentiated using the PCR-RFLP approach, but $\mathrm{A}, \mathrm{B}$ and $\mathrm{C}$ haplotypes differ from each other by one SNP and were found only in Cx. pipiens $\mathrm{f}$. pipiens. This made it possible to combine them into haplogroup A (Shaikevich and Zakharov, 2014). Similarly, haplotypes $\mathrm{E}$ and E1 are close relatives and were found predominantly in Cx. quinquefasciatus, and we put them together into haplogroup E (Shaikevich et al., 2016). Monomorphic haplotype D was found in Cx. pipiens $\mathrm{f}$. molestus (Shaikevich and Zakharov, 2010; Shaikevich and Zakharov, 2014; Shaikevich et al., 2016).

At the same time, $C x$. torrentium have numerous haplotypes, and each studied individual has a unique haplotype (Shaikevich and Zakharov, 2010). The data show a considerable decrease in mtDNA polymorphism in the Wolbachia-infected population of Cx. pipiens compared with uninfected Cx. torrentium (Table 1 ). These data correspond to the observations of Rasgon et al. (2006) that Wolbachia-uninfected Culex mosquitoes have high mtDNA diversity. Polymorphism in the nuclear sequence (ITS2) was similar in infected and uninfected Cx. pipiens (Rasgon et al., 2006; Dumas et al., 2016), as well as in infected $C x$. pipiens and uninfected Cx. torrentium (Shaikevich and Zakharov, 2010). This suggests that the reduction of mitochondrial polymorphism is more likely to be due to the selective sweep of the Wolbachia-infected haplotype than to a passage of $C x$. pipiens through a bottleneck before the spread around the world.

Table 1. Diversity in Wolbachia-infected and uninfected Culex based on COI sequence polymorphism

\begin{tabular}{l|c|c|c|c|c|c}
\hline \multicolumn{1}{c|}{ Species } & Wolbachia & $\mathrm{N}$ & $\mathrm{h}$ & $\mathrm{S}$ & $\mathrm{Pi}$ & $\mathrm{K}$ \\
\hline $\begin{array}{l}\text { Cx.pipiens } \\
\text { f.pipiens }\end{array}$ & $98 \%$ & 10 & 3 & 3 & 0.0008 & 1.24 \\
\hline $\begin{array}{l}\text { Cx.pipiens } \\
\text { f.molestus }\end{array}$ & $100 \%$ & 10 & 0 & 0 & 0 & 0 \\
\hline Cx.torrentium & $0 \%$ & 10 & 10 & 21 & 0.00346 & 5.4 \\
\hline
\end{tabular}

N. - studied specimens, $\mathrm{h}$ - number of haplotypes, $\mathrm{S}$ - number of polymorphic sites, $\mathrm{Pi}$ - nucleotide diversity, $\mathrm{K}$ - average number of nucleotide differences

The influence of infection on the fitness of $C x$. pipiens is difficult to assess, since the infection rate in natural populations of this species is almost $100 \%$ (Shaikevich and Zakharov, 2010; Atyame et al., 2011; Dumas et al., 2016; Shaikevich et al., 2016). However, in laboratory experiments it was demonstrated that Wolbachia-infected $C x$. quinquefasciatus females develop and lay their eggs earlier compared to the cured mosquitoes. This effect and induction of CI were found to be reproductively advantageous to infected females, compared to aposymbiotic ones (Almeida et al., 2011).
WOLBACHIA VARIABILITY AND

ASSOCIATION WITH MTDNA IN CULEX PIPIENS

Wolbachia from Culex pipiens s.l. isolates collected in different geographical regions have identical MLST profiles and wsp sequences (Shaikevich et al., 2019). However, detailed analysis that included some other genes, coding proteins with ankyrin (ANK) domains (Atyame et al., 2011), revealed the genogeographic specificity of bacterial strains (Dumas et al., 2013; Shaikevich et al., 2016). Wolbachia induces cytoplasmic incompatibility (CI) in Cx. pipiens (Ghelelovitch, 1952; Laven, 1967; Atyame et al., 2014). The unidirectional CI phenomenon is embryonic mortality of offspring when an infected male mates with an uninfected female; the bidirectional $\mathrm{CI}$ is when the male and female are infected with different strains (Yen and Barr, 1971; Werren et al., 2008; Atyame et al., 2014). Five genetically different groups of Wolbachia $(w$ Pip) show different incompatibility status (Atyame et al., 2014).

In Russia, three different $w$ Pip groups were identified in Culex pipiens s.l. (Shaikevich and Zakharov, 2014). More haplotypes and $w$ Pip groups were detected in $C x$. pipiens s.l. populations in the Mediterranean region (Shaikevich et al., 2016). Culex mtDNA of $580 \mathrm{spec}-$ imens from 35 geographical populations was genotyped using a series of specific PCR-RFLP assays (Shaikevich et al., 2016). The association between the COI haplogroup and the $w$ Pip group shows the co-transmission of cytoplasmic components (Table 2).

A strong association was observed between the COI D haplotype, as well as the $\mathrm{A}$ and $\mathrm{E}$ haplogroups and $w$ Pip groups and taxa (Chi-square $=732.71$, d.f. $=$ $8, P<0.0001)$ in 35 distinct geographical populations of the Culex pipiens complex. Haplogroup A and infection with $w$ PipII and $w$ PipIII appear to be typical for $C x$. pipiens $\mathrm{f}$. pipiens (Table 3). Haplotype $\mathrm{D}$ and infection with $w$ PipIV are characteristic of the f. molestus, while haplogroup E, characteristic of Cx. quinquefasciatus, was correlated with $w$ PipI (Shaikevich et al., 2016). Infection of $C x$. quinquefasciatus with bacteria of the $w$ Pip-I group and $C x$. pipiens (f. pipiens) with bacteria of the $w$ Pip-II and $w$ Pip-III groups was found in a study of 823 mosquitoes from around the world with high statistical reliability (Fisher exact test, $\mathrm{P}<10^{-4}$ ) (Dumas et al., 2013).

Hybridization events in $C x$. pipiens populations in coastal regions of Southern Europe and North Africa lead to the mixing of cytoplasmic components among taxa (Shaikevich and Vinogradova, 2014; Shaikevich et al., 2016). Therefore, the pattern of mtDNA variation reflects the evolutionary history of $w$ Pip infection rather than of the mosquito taxa in the Mediterranean region (Dumas et al., 2013). Nevertheless, a statistically significant correlation between the COI haplogroup and taxa has been observed also in sympatric populations after 
Table 2. The association between mtDNA of Culex pipiens s.I. and type of bacteria Wolbachia

\begin{tabular}{|c|c|c|c|c|}
\hline Sampling place & $\mathrm{N}$ & COI type** & $\begin{array}{l}\text { wPip*** } \\
\text { group }\end{array}$ & References \\
\hline Russia, Krasnodar & 6 & A & II & Shaikevich et al., 2016 \\
\hline Russia, Mos. obl & 16 & A & ॥ & Shaikevich and Zakharov, 2014 \\
\hline Russia, Moscow* & 20 & $\mathrm{D}$ & IV & Shaikevich et al., 2016 \\
\hline Russia, S-Peterburg1* & 8 & D & IV & Shaikevich et al., 2016 \\
\hline Russia, S-Peterburg2* & 7 & D & IV & Shaikevich et al., 2016 \\
\hline Russia, Ekaterinburg* & 6 & D & IV & Duma et al., 2013 \\
\hline Russia, Tomsk* & 9 & $\mathrm{D}$ & IV & Duma et al., 2013 \\
\hline Russia, Volgograd & 12 & A & II & Shaikevich et al., 2016 \\
\hline Russia, Volgograd & 7 & A & III & Shaikevich and Zakharov, 2014 \\
\hline Russia, Volgograd* & 8 & $\mathrm{D}$ & IV & Shaikevich et al., 2016 \\
\hline Kazakhstan, Almaty & 21 & $\mathrm{D}$ & IV & Shaikevich and Zakharov, 2014 \\
\hline Tunis, Nefza & 16 & D & IV & Shaikevich et al., 2016 \\
\hline Tunis, Tabarka & 12 & $\mathrm{D}$ & IV & Shaikevich et al., 2016 \\
\hline Morocco, Tanger & 1 & $\mathrm{E}$ & I & Shaikevich et al., 2016 \\
\hline Morocco, Casablanca & 2 & A & II & Shaikevich et al., 2016 \\
\hline Greece, Kos & 24 & E & 1 & Shaikevich \& Vinogradova, 2013 \\
\hline Italy, Piedmont & 18 & $\mathrm{D}$ & IV & Shaikevich et al., 2016 \\
\hline Portugal, Comporta & 4 & $\mathrm{E}$ & I & Shaikevich et al., 2016 \\
\hline Portugal, Comporta & 6 & $\mathrm{E}$ & I & Shaikevich et al., 2016 \\
\hline Germany, Berlin* & 4 & $\mathrm{D}$ & IV & Shaikevich et al., 2016 \\
\hline Germany, Berlin & 9 & A & II & Shaikevich et al., 2016 \\
\hline Germany, Hannover* & 1 & D & IV & Shaikevich et al., 2016 \\
\hline Germany, Hannover & 17 & A & ॥ & Shaikevich et al., 2016 \\
\hline France, Prades-le-Lez 1 & 16 & $A$ & ॥ & Shaikevich et al., 2016 \\
\hline France, Prades-le-Lez 2 & 22 & A & II & Shaikevich et al., 2016 \\
\hline France, Saint-Nazaire de Pezan & 12 & A & ॥ & Shaikevich et al., 2016 \\
\hline T7 strain, France,Montpellier & 11 & A & ॥ & Shaikevich et al., 2016 \\
\hline Pondicherry, India & 23 & E & 1 & Shaikevich and Zakharov, 2014 \\
\hline Hyderabad, India & 20 & E & 1 & Shaikevich and Zakharov, 2014 \\
\hline Total & 338 & & & \\
\hline
\end{tabular}

the confirmation of $C x$. pipiens taxonomy status by microsatellite (CQ11) assay (Shaikevich et al., 2016). Fixed nucleotide substitutions in the COI gene that can distinguish the complex members were found in allopatric $C x$. pipiens $\mathrm{f}$. pipiens and $\mathrm{f}$. molestus populations in a temperate climate, as well as between temperate $C x$. pipiens and tropical Cx. quinquefasciatus (Shaikevich and Zakharov, 2010; Danabalan et al., 2012; Gunay et al., 2015; Shai- kevich et al., 2016). The existing subspecies-specific pattern of mtDNA haplotypes and its association with the $w$ Pip group among Wolbachia-infected populations of mosquitoes $C x$. pipiens s.l. suggest that the infection of mosquitoes with the endosymbiotic bacteria occurred earlier than the divergence of mitochondrial DNA and taxa. Probably, bacterial infection played a key role in subspecies formation, since it is known that $w$ Pip induc- 
Table 3. Partitioning of $\mathrm{COI}$ haplotypes between $\mathrm{Cx}$. pipiens taxa

\begin{tabular}{l|c|c|c|c|c}
\hline \multirow{2}{*}{ COI type } & \multirow{2}{*}{$\mathrm{N}$} & \multicolumn{3}{|c}{ Cx. pipiens taxa } \\
\cline { 3 - 6 } & & $\begin{array}{c}\text { Cx. pipiens f. } \\
\text { pipiens }\end{array}$ & $\begin{array}{c}\text { Cx. pipiens f. } \\
\text { molestus }\end{array}$ & Cx. quinquefasciatus & Cx. pipiens/Cx. quinquefasciatus hybrid \\
\hline Group A & 222 & $201(91 \%)$ & $11(5 \%)$ & 0 & $10^{*}(4 \%)$ \\
\hline D & 220 & $20(9 \%)$ & $179(81 \%)$ & 0 & $21^{*}(10 \%)$ \\
\hline Group E & 125 & $10^{*}$ & $10^{*}$ & 43 & 0 \\
\hline
\end{tabular}

* only in Mediterranean population

es different cytoplasmic incompatibility patterns in $C x$. pipiens (Atyame et al., 2014; Altinli et al., 2018).

\section{Wolbachia variability and association with mtDNA in Aedes albopictus}

Two Wolbachia strains, wAlbA and wAlbB, coinfect nearly all studied Ae. albopictus. Wolbachia-infected Ae. albopictus females were observed to have increased longevity, greater oviposition rates, and higher egg hatch rates and an increased fecundity (Dobson et al., 2002). Wolbachia induces cytoplasmic incompatibility in Ae. albopictus (Sinkins et al., 1995; Dobson et al., 2004). Most populations are naturally infected with both strains, suggesting that superinfection is common in the field-collected Ae. albopictus worldwide (Armbruster et al., 2003; Ahmad et al., 2017). The frequency of Wolbachia infection in most Ae. albopictus populations tends to $100 \%$, but it varies in certain populations. On the Russian Caucasus coast, both $w$ AlbA and $w$ AlbB strains were found in Ae. albopictus, collected in 2011, 2012, 2013 and 2016 (Shaikevich et al., 2018). Of the 411 studied individuals, $56 \%$ were Wolbachia-positive. Among 234 infected specimens, 3 variants of infection were found: rare strain $w \mathrm{AlbA}(1.7 \%)$, common strain $w \mathrm{AlbB}$ (78.6\%), and superinfection with both strains $w$ AlbA and $w$ AlbB (19.7\%); 177 specimens were not infected. Among them, 159 mosquitoes were Wolbachia-free in one collection in 2016 from Dagomys. Good PCR results for the other mosquito genes ruled out the poor quality of mosquito DNA in this collection (Shaikevich et al., 2018). The frequency of Wolbachia infections was recently investigated in $14 \mathrm{Ae}$. albopictus populations in China (Guo et al., 2018). Most positive individuals were infected with both the $w \mathrm{AlbA}$ and $w \mathrm{AlbB}$ strains of Wolbachia. The infection rate in eight populations was $100 \%$. But, in two of the Ae. albopictus populations, only $50 \%$ and $60 \%$ of individuals were infected with Wolbahia (Guo et al., 2018).

In contrast to Culex pipiens, high mtDNA polymorphism is observed in Ae. albopictus populations, especially in the areas of the native range of the species in Southeast Asia (Poretta et al., 2012; Fang et al., 2018; Ruling et al., 2018). Some previous studies of Ae. albop- ictus populations supposed a low genetic diversity in mitochondrial markers (Ambrurster et al., 2003 and reference therein). This was later refuted (Poretta et al., 2012; Battaglia et al., 2016): the reasons for the low genetic diversity were that 1 ) in the first studies, most Ae. albopictus mitochondrial DNA surveys were restricted to short segments of the COI and/or NADH dehydrogenase subunit 5 (ND5) genes; 2) laboratory stocks or sibling eggs were included in these studies. Hundreds of haplotypes were identified after the analyses of almost the entire COI gene ( $90 \%$ of the entire COI gene in length) both in native and colonized areas (Porreta et al., 2012; Zhong et al., 2013; Giordano et al., 2019; Hu et al., 2020; Zé-Zé et al., 2020). No co-evolution of mtDNA haplotypes and $w$ Alb strains or any loss of mtDNA diversity in infected Ae. albopictus individuals was revealed (Minard et al., 2017; Guo et al., 2018).

If one compares Wolbachia-infected Ae. albopictus and uninfected representatives of cryptic species, the latter demonstrate greater variability of mtDNA in China (Guo et al., 2018), but not in Vietnam (Minard et al., 2017). In Southeast Asia, sympatric cryptic species have been identified in the Ae. albopictus subgroup (Minard et al., 2017; Guo et al., 2018). Ae. albopictus sibling species are combined in the literature as members of the Scutellaris group and Ae. albopictus subgroup. These species have very similar morphological characteristics, especially at the larval and adult (females) stages. Furthermore, although some of the species of this subgroup have different ecological niches, some of them are found in sympatry (Minard et al., 2017 and the reference therein). Contrary to Ae. albopictus, the cryptic species did not harbor any Wolbachia infection in Vietnam. However, individuals of cryptic species presented similar haplotype diversity relative to Ae. albopictus in Vietnam (Minard et al., 2017). Another pattern was observed in the cryptic species in China (Guo et al., 2018). Wolbachia was rare but was present in populations of cryptic species in China. A total of 47 COI haplotypes were detected in 129 Ae. albopictus mosquitoes and 10 haplotypes derived from 11 specimens of cryptic species, indicating significantly higher genetic diversity found in cryptic species populations than in Ae. albopictus populations in China (Guo et al., 2018). 


\section{Conclusion}

In the Culex pipiens complex and in the cryptic species Cx. torrentium, uninfected individuals show higher mitochondrial diversity than infected ones (Rasgon et al., 2006; Shaikevich and Zakharov, 2010; Atyame et al., 2011; Dumas et al., 2016). Indeed, non-infected populations of $C x$. pipiens harbored a higher ancestral mitochondrial diversity (Rasgon et al., 2006). The initial $w$ Pip sweep through $C x$. pipiens s.l. is estimated to have occured 20,000 years ago (Dumas et al., 2016) or up to 47,000 years ago (Rasgon et al., 2006). This indicates that $w$ Pip invasion in the $C x$. pipiens complex was recent and rapid. The invasion of the $w$ Pip ancestor resulted in an indirect selective mitochondrial sweep due to CI, which led to the loss of mtDNA variation within $C x$. pipiens populations (Rasgon et al., 2006; Dumas et al., 2016).

Such mitochondrial sweep was not supported in Ae. albopictus, as no significant differences in the haplotype diversities were observed between Ae. albopictus and the cryptic species (Minard et al., 2017). High genetic diversity is typical for individuals of Ae. albopictus infected with $w$ Alb in the native range (Ruling et al., 2018). The diversity of mtDNA in Ae. albopictus is reduced in some invasive populations due to the founder effect, unrelated to Wolbachia infection. The fact that individuals with different mitochondrial haplotypes are infected with the same $w$ AlbA and $w$ AlbB strains indicates that mtDNA diversity arose after the initial infection. Moreover, recombination between primarily monophyletic wAlbA and $w$ AlbB strains was observed in Ae. albopictus (Shaikevich et al., 2019). These facts, together with the wide diversity of mtDNA haplotypes, indicate an ancient infection in Ae. albopictus.

\section{Ladybirds}

Beetles of the Adalia genus (Coleoptera: Coccinellidae) have been the subject of research in the field of ecological genetics for a long time due to their wide distribution and easily registered morphological polymorphism. In the 2000s, the study of their molecular polymorphism began. When analyzing the nucleotide diversity of the COI mitochondrial gene in Adalia bipunctata, 17 mitotypes were described (Shaikevich and Zakharov, 2015). Most of them differ by 1-4 nucleotides; however, two mitotypes, $\mathrm{H} 9$ and H10, differ from the others at the interspecies level (Schulenburg et al., 2002; Shaikevich and Zakharov, 2015); they may have been included in the gene pool of A. bipunctata as a result of ancient hybridization with an unknown (extinct?) species.

Studying the hybridization of beetles of different populations, Lus (1947) discovered the existence of females giving only female offspring. This feature turned out to be an inherited trait passed strictly through the female line. Forty-five years after the discovery of this phenomenon, its nature was deciphered - it turned out that the one-sex female offspring was the result of the death of male embryos, which is caused by symbiotic bacteria living in the cytoplasm (Werren et al., 1994). DNA analysis allowed identification of these bacteria. The same effect, the death of male offspring, is caused by three different bacteria: Rickettsia, Wolbachia and Spiroplasma. Beetles infected with different bacteria may be present in the same population (Majerus et al., 2000).

Since the same population of ladybirds may contain individuals infected with different female-transmitted bacteria, the relationship between the presence of a particular bacterium and the host mitotype has been studied. In the work by Schulenburg et al. (2002) (with the participation of one of the authors of this article), a correlation between bacterial infection and the mtDNA type was shown.

Rickettsia was found in individuals of mitotypes $\mathrm{H} 9$ and $\mathrm{H} 10$ (one individual had mitotype H7); Wolbachia - in individuals of mitotypes H1(one strain of this bacterium), H3, H6, H8 (another strain); Spiroplasma in individuals of mitotypes $\mathrm{H} 1, \mathrm{H} 2$. In this study, beetles from populations of Great Britain, Germany, Denmark and Russia (the majority of individuals from St. Petersburg and Moscow) were studied. The authors continued this research by using material from a broader geographical origin: from Moscow, St. Petersburg, Arkhangelsk, Karelia, Buryatia, and other places (Zakharov and Shaikevich, 2011; Shaikevich and Zakharov, 2015).

Rickettsia was found in individuals of the H10 mitotype (a total of 19 individuals from 3 populations); Spiroplasma - in 19 individuals from 2 populations with the $\mathrm{H} 1, \mathrm{H} 2, \mathrm{H} 3, \mathrm{H} 11$ and H17 mitotypes. It should be noted that the $\mathrm{H} 2, \mathrm{H} 3, \mathrm{H} 11$ and $\mathrm{H} 17$ mitotypes are related to the H1 mitotype and differ from it by one nucleotide each.

What can be concluded from the above data?

The wide geographical distribution of two symbiotic bacteria (Rickettsia and Spiroplasma), with a clear "coupling" of infection with certain mitotypes, indicates that infection is a rare event and it occurred long ago, at least before the end of the last ice age. The prescription of the act of infection of ladybirds with Spiroplasma bacteria is also indicated by the fact that the bacterium occurs in individuals with several, but related mitotypes - that is, mutations-replacements of single nucleotides that led to the appearance of the 2, 3, 11 and 17 mitotypes occurred after the act of infection. This is also evidenced by the presence of Rickettsia in a ladybird (mitotype H10) from Buryatia, where, as is known, there is a special subspecies, A. bipunctata fasciatopunctata; that is, differentiation into subspecies occurred after infection with Rickettsia of the ancestral population of beetles.

The horizontal transfer of bacteria, if it occurs, is rare. It can only be described by a single case of finding 
Rickettsia in a beetle not of the H9 or H10 mitotypes, but of the H7 mitotype, which is not related to the first two (Schulenburg et al., 2002).

In individuals of another species, A. decempunctata, only Rickettsia (Schulenburg et al., 2001; Zakharov and Shaikevich, 2001) was found from symbiotic bacteria. Eight mitotypes were identified in this species (Shaikevich et al., 2019), but no association of mitotypes and Rickettsia infection was found.

\section{Symbionts of different arthropods}

The first or one of the first studies to demonstrate the relationship of a symbiont with a specific variant of mtDNA was a study that examined the diversity of mtDNA in individuals of $D$. simulans infected and uninfected with Wolbachia. Individuals infected with Wolbachia from populations from California (USA), Canada, Italy and South Africa all had the same mitotype (B), while among the uninfected individuals, two other mitotypes were identified along with mitotype B (Hale and Hoffmann, 1990).

The fast (within a few years) spread of Wolbachia, which causes the phenomenon of cytoplasmic incompatibility, was shown in the study of populations of this species of Drosophila from California. Infected flies had a single mitotype. Uninfected individuals from the same populations showed high mtDNA polymorphism (Turelli et al., 1992).

Ilinsky (2013) studied a large number of Drosophila melanogaster lines of different geographical origin and revealed two main mitotype clades: $M$ and $S$, each being strictly associated with one of the two major Wolbachia groups, MEL and CS, respectively (see also Bykov et al., 2019). No evidence of horizontal transmission of Wolbachia between maternal lineages has been found.

In the terrestrial crustacean Armadillidium vulgare, 11 mitotypes were detected; in individuals from different populations infected with Wolbachia, which causes the feminization of genetically male individuals, only 2 of the 11 were found. These two mitotypes, Av1 and Av2, were closely related (Rigaud et al., 1999).

Populations of Acraea encedon and A. encedana butterflies consist of individuals infected with Wolbachia, which in these species causes the death of male offspring and uninfected individuals. The first species is infected with two strains of Wolbachia; the second, with one species. Nine mitotypes are variously represented among infected and uninfected individuals. The UG mitotype is found among infected individuals of both species in populations of Ghana, Tanzania, Uganda and Zimbabwe, as well as among uninfected individuals of both species from Uganda. The TZ mitotype is found only in infected A.encedon from Tanzania; the remaining seven mitotypes were found only among uninfected individu- als of A.encedon. It can be concluded that the symbiont is maintained in populations of A. encedon linked to one mitotype and the same pair of genomes as a result of introgression during interspecific hybridization was transferred to the gene pool of the second species (Jiggins, 2003).

\section{General conclusion}

The materials presented in this article show a wide spread of quasi-linkage of cytoplasmic genomes among insects and other arthropods. Since the transfer of intracellular symbionts usually occurs through female gametes, the phenomenon discussed should occur in all cases where there is no or very rare horizontal transmission of the symbiont. A discussion of the consequences of quasilinkage of mitochondrial genomes and cytoplasmic symbionts is given in the article by Johnstone and Hurst (1996), see also Perlman et al. (2015).

What are the biological consequences of this phenomenon?

The first obvious consequence is the spread of a special mitotype in the population, which itself does not have increased selective value. The symbiont acts as a "driver" and in cases when the symbiont spreads in the population, the "linked" mitotype also spreads. There are two mechanisms of increasing the frequency of symbiont-infected individuals in the population: the symbiont manipulates host reproduction, causing the domination of infected individuals in the offspring, and the symbiont increases the fitness of infected individuals in comparison with uninfected ones (as a result of increased resistance to parasites, increased lifespan, etc.). The distribution of Wolbachia in California populations of Drosophila simulans and the simultaneous distribution of a special mitotype, not previously encountered in these populations, illustrates the phenomenon (Turelli et al., 1992).

It is possible to assume the coadaptation of the "linked" cytoplasmic genomes - both the genome of a symbiont that found itself in a new cell environment and the mitochondrial genome, since the presence of a large number of bacteria in the cytoplasm must affect the energy balance of the cell. It is difficult to detect such coadaptation. Possibly an increase in the frequency of mutations in mtDNA in Wolbachia-infected lines may reflect the process of coadaptation of cytoplasmic genomes (Shoemaker et al., 2004).

Finally, species of the Lepidoptera order have heterogametic female sex. As a result, three genomes are linked - the W chromosome, mtDNA, and the symbiont genome. The $\mathrm{W}$ chromosome must also be involved in the coadaptation process along with the other two genomes. 


\section{References}

Ahmad, N. A., Vythilingam, I., Lim, Y.A. L., Zabari, N. Z. A. M., and Lee, H. L. 2017. Detection of Wolbachia in Aedes albopictus and their effects on chikungunya virus. American Journal Tropical Medicine and Hygiene 96(1):148-156. https://doi.org/10.4269/ajtmh.16-0516

Almeida, F., Moura, A.S., Cardoso, A. F., Winter, C. E., Bijovsky, A. T., and Suesdek, L. 2011. Effects of Wolbachia on fitness of Culex quinquefasciatus (Diptera; Culicidae). Infection Genetic and Evolution 11(8):2138-2143. https:// doi.org/10.1016/j.meegid.2011.08.022

Altinli, M., Gunay, F., Alten, B., Weill, M., and Sicard, M. 2018. Wolbachia diversity and cytoplasmic incompatibility patterns in Culex pipiens populations in Turkey. Parasites and Vectors 11(1):198. https://doi.org/10.1186/s13071018-2777-9

Armbruster, P., Damsky, W. E., Giordano, R., Birungi, J., Munstermann, L. E., and Conn, J. E. 2003. Infection of newand old-world Aedes albopictus (Diptera: Culicidae) by the intracellular parasite Wolbachia: implications for host mitochondrial DNA evolution. Journal of Medical Entomology 40:356-360. https://doi.org/10.1603/00222585-40.3.356

Atyame, C. M., Delsuc, F., Pasteur, N., Weill, M., and Duron, O. 2011. Diversification of Wolbachia endosymbiont in the Culex pipiens mosquito. Molecular Biolology and Evolution 28:2761-2772. https://doi.org/10.1093/molbev/msr083

Atyame, C. M., Labbé, P., Dumas, E., Milesi, P., Charlat, S., Fort, Ph., and Weil, M. 2014. Wolbachia divergence and the evolution of cytoplasmic incompatibility in Culex pipiens. PLoS One 9(1):e87336. https://doi.org/10.1371/journal.pone.0087336

Battaglia, V., Gabrieli, P., Brandini, S., Capodiferro, M. R., Javier, P. A., Chen, X. G., Achilli, A., Semino, O., Gomulski, L. M., Malacrida, A. R., Gasperi, G., Torroni, A., and Olivieri, A. 2016. The worldwide spread of the tiger mosquito as revealed by mitogenome haplogroup diversity. Frontiers in Genetics 7:208. https://doi.org/10.3389/fgene.2016.00208

Bykov, R. A., Yudina, M. A., Gruntenko, N. E., Zakharov, I. K., Voloshina, M. A., Melashchenko, E.S., Danilova, M. V., Mazunin, I. O., and Ilinsky, Y. I. 2019. Prevalence and genetic diversity of Wolbachia endosymbiont and mtDNA in Palearctic populations of Drosophila melanogaster. BMC Evolutionary Biology 19(Suppl 1):48. https://doi. org/10.1186/s12862-019-1372-9

Danabalan, R., Ponsonby, D. J., and Linton, Y.-M. 2012. A critical assessment of available molecular identification tools for determining the status of Culex pipiens S. L. in the United Kingdom. Journal of American Mosquito Control Association 28(Suppl 4):68-74. https://doi. org/10.2987/8756-971X-28.0.68

Dobson, S. L., Marsland, E. J., and Rattanadechakul, W. 2002. Mutualistic Wolbachia infection in Aedes albopictus: accelerating cytoplasmic drive. Genetics 160:1087-1094.

Dobson, S. L., Rattanadechakul, W., and Marsland, E. J. 2004. Fitness advantage and cytoplasmic incompatibility in Wolbachia single- and superinfected Aedes albopictus. Heredity 93(2):135-142. https://doi.org/10.1038/ sj.hdy. 6800458

Dumas, E., Atyame, C. M., Milesi, P., Fonseca, D. M., Shaikevich, E. V., Unal, S., Makoundou, P., Weil, M., and Duron, O. 2013. Population structure of Wolbachia and cytoplasmic introgression in a complex of mosquito species. BMC Evolutionary Biology 13:183. https://doi. org/10.1186/1471-2148-13-181

Dumas, E., Atyame, C. M., Malcolm, C. A., Le Goff, G., Unal, S., Makoundou, P., Pasteur, N., Weil, M., and Duron, O.
2016. Molecular data reveal a cryptic species within the Culex pipiens mosquito complex. Insect Molecular Biology 25:800-809. https://doi.org/10.1111/imb.12264

Fang, Y., Zhang, J., Wu, R., Xue, B., Qian, Q., and Gao, B. 2018. Genetic polymorphism study on Aedes albopictus of different geographical regions based on DNA barcoding. Biomed Research International 1501430. https://doi. org/10.1155/2018/1501430

Fedorova, M. V. and Shaikevich, E. V. 2007. Morphological and molecular-genetic differences between the adults of mosquitoes Culex torrentium Martini and Culex pipiens L. from Moscow Province. Entomological Review 87:127135. https://doi.org/10.1134/S0013873807020017

Ghelelovitch, S. 1952. Sur le diterminisme genttique de la sterilitc dans le croisement entre differentes souches de Culex autogenicus Roubaud. C. R. Acad. Sci. Paris 234:2386-2388.

Giordano, B., Gasparotto, A., Liang, P., Nelder, M., Russell, C., and Hunter, F. 2019. Discovery of an Aedes (Stegomyia) albopictus population and first records of Aedes (Stegomyia) aegypti in Canada. Medical and Veterinary Entomology 34(1):10-16. https://doi.org/10.1111/mve.12408

Gomes, B., Sousa, C. A., Novo, M. T., Freitas, F. B., Alves, R., Corte-Real, A. R., Salgueiro, P., Donnelly, M. J., Almeida, A.P. G., and Pinto, J. 2009. Asymmetric introgression between sympatric molestus and pipiens forms of Culex pipiens (Diptera: Culicidae) in Comporta region, Portugal. BMC Evolutionary Biology 9:262. https://doi. org/10.1186/1471-2148-9-262

Gunay, F., Alten, B., Simsek, F., Aldemir, A., and Linton, Y.-M. 2015. Barcoding Turkish Culex mosquitoes to facilitate arbovirus vector incrimination studies reveals hidden diversity and new potential vectors. Acta Tropica 143:112120. https://doi.org/10.1016/j.actatropica.2014.10.013

Guo, Y., Song, Z., Luo, L., Wang, Q., Zhou, G., Yang, D., Zhong, D., and Zheng, X. 2018. Molecular evidence for new sympatric cryptic species of Aedes albopictus (Diptera: Culicidae) in China: A new threat from Aedes albopictus subgroup? Parasites and Vectors 11:228. https://doi. org/10.1186/s13071-018-2814-8

Hale, L. R. and Hoffmann, A. A. 1990. Mitochondrial DNA polymorphism and cytoplasmic incompatibility in natural populations of Drosophila simulans. Evolution 44:1383-1386. https://doi.org/10.1111/j.1558-5646.1990.tb05241.x

Harbach, R. 2012. Culex pipiens: Species versus species complex - taxonomic history and perspective. Journal of the American Mosquito Control Association 28:10-23. https:// doi.org/10.2987/8756-971X-28.4.10

Hu, Y., Xi, Z., Liu, X., Wang, J., Guo, Y., Ren, D., Wu, H., Wang, X., Chen, B., and Liu, Q. 2020. Identification and molecular characterization of Wolbachia strains in natural populations of Aedes albopictus in China. Parasites and Vectors 13(1):28. https://doi.org/10.1186/s13071-020-3899-4

Ilinsky, Y. 2013. Coevolution of Drosophila melanogaster mtDNA and Wolbachia genotypes. PLOS ONE 8(1):e54373. https://doi.org/10.1371/journal.pone.0054373

Jiggins, F. M. 2003. Male-killing Wolbachia and mitochondrial DNA: selective sweeps, hybrid introgression and parasite population dynamics. Genetics 164:5-12.

Johnstone, R. A. and Hurst, G. D. D. 1996. Maternally inherited male-killing microorganisms may confound interpretation of mitochondrial DNA variability. Biological Journal of the Linnean Society 58:453-470.

Laven, H. 1967. Eradication of Culex pipiens fatigans through cytoplasmic incompatibility. Nature 216:383-384. https://doi.org/10.1038/216383a0

Lederberg, J. 1952. Cell genetics and hereditary symbionts. Physiological Review 32:403-430. https://doi.org/10.1152/ physrev.1952.32.4.403 
Majerus, M.E. N., Schulenburg J.H.G., and Zakharov, I.A. 2000. Multiple causes of male-killing in a single sample of the two-spot ladybird, Adalia bipunctata (Coleoptera: Coccinellidae) from Moscow. Heredity 84:605-609. https://doi.org/10.1046/j.1365-2540.2000.00710.x

Minard, G., Van, V., Tran, F. H., Melaun, C., Klimpel, S., Koch, L. K., Kim, K. L. H. K., Thuy, T.H.T.T., Ngoc, H.T., Potier, P., Mavingui, P., and Moro, C. V. 2017. Identification of sympatric cryptic species of Aedes albopictus subgroup in Vietnam: new perspectives in phylosymbiosis of insect vector. Parasit Vectors 10:276. https://doi. org/10.1186/s13071-017-2202-9

Perlman, S. J., Hodson, C. N., Hamilton, P. T., Opit, G. P., and Gowen, B. E. 2015. Maternal transmission, sex ratio distortion, and mitochondria. Proceedings of the National Academy of Sciences USA 112(33):10162-10168. https:// doi.org/10.1073/pnas.1421391112

Porretta, D., Mastrantonio, V., Bellini, R., Somboon, P., and Urbanelli, S. 2012. Glacial history of a modern invader: Phylogeography and species distribution modelling of the Asian tiger mosquito Aedes albopictus. PLOS ONE 7(9):e44515. https://doi.org/10.1371/journal.pone.0044515

Preer, J. R. 1971. Extrachromosomal inheritance: hereditary symbionts, mitochondria, chloroplasts. The Annual Review of Genetics 5:361-406. https://doi.org/10.1146/annurev.ge.05.120171.002045

Provorov, N. A. and Tikhonovich, I. A. 2014. Super-species genetic systems. Zhurnal obshcher biologii 75(4):247-260. (In Russian)

Rasgon, J. L., Cornel, A. J., and Scott, T. W. 2006. Evolutionary history of a mosquito endosymbiont revealed through mitochondrial hitchhiking. Proceedings of the Royal Society B: Biological Sciences 273:1603-1611. https://doi. org/10.1098/rspb.2006.3493

Rigaud, T., Bouchon, D., Souty-Grosset, C., and Raimond, R. 1999. Mitochondrial DNA polymorphism, sex ratio distorters and population genetics in the isopod Armadillidium vulgare. Genetics 152:1669-1677.

Ruiling, Z., Tongkai, L., Dezhen, M., and Zhong, Z. 2018. Genetic characters of the globally spread tiger mosquito, Aedes albopictus (Diptera, Culicidae): implications from mitochondrial gene COI. Journal of Vector Ecology 43:8997. https://doi.org/10.1111/jvec.12287

Schulenburg, J. H., Habig, M., Sloggett, J. J., Webberley, K. M., Bertrand, D., Hurst, G. D., and Majerus, M. E. 2001. Incidence of male-killing Rickettsia spp. (a-proteobacteria) in the ten-spot ladybird beetle Adalia decempunctata $\mathrm{L}$. (Coleoptera: Coccinellidae). Applied and Environmental Microbiology 67:270-277. https://doi.org/10.1128/ AEM.67.1.270-277.2001

Schulenburg, J. H. G., Hurst, G. D. D., Tetzlaff, D., Booth, G. E., Zakharov, I. A., and Majerus, M. E. 2002. History of infection with different male-killing bacteria in the two-spot ladybird beetle Adalia bipunctata revealed through mitochondrial DNA sequence analysis. Genetics 160:10751086.

Shaikevich, E. V., Fedorova, M. V., and Vinogradova, E. B. 2006. DNA diagnostics of representatives of the Culex pipiens complex (Culicidae, Diptera) from Russia. Proceeding of I Conference on Bloodsucking Insects 227-228.

Shaikevich, E. V. 2007. PCR-RFLP of the COI gene reliably differentiates $C x$. pipiens, $C x$. pipiens form molestus and $C X$. torrentium of the Pipiens Complex. European Mosquito Bulletin 23:25-30.

Shaikevich, E. V. 2009. Identification of Culex mosquitoes (Diptera, Culicidae) by the restriction assay of amplification products. Meditsinskaia parazitologiia i parazitarnye bolezni 3:28-32. (In Russian)
Shaikevich, E. V. and Zakharov, I. A. 2010. Polymorphism of mitochondrial $\mathrm{COI}$ and nuclear ribosomal ITS2 in Culex pipiens complex and in Culex torrentium (Diptera, Culicidae). Comparative Cytogenetics 4(2):161-174. https://doi. org/10.3897/compcytogen.v4i2.45

Shaikevich, E. V. and Zakharov, I. A. 2014. Coevolution of symbiotic bacteria Wolbachia and host mtDNA in Russian populations of the Culex pipiens mosquito complex. Russian Journal of Genetics 50(11):1234-1237. https://doi. org/10.1134/S1022795414110131

Shaikevich, E. V. and Zakharov, I. A. 2015. Biodiversity in geographically remote natural populations of Adalia ladybirds (Coleoptera: Coccinellidae), pp. 205-226 in C. Stack (ed.) Beetles: biodiversity, ecology and role in the environment. Nova Science Publishers.

Shaikevich, E., Vinogradova, E., Boatour, A., and Almeida, P. 2016. Genetic diversity of Culex pipiens mosquitoes in distinct populations from Europe. Contribution of $C X$. quinquefasciatus in Mediterranean populations. Parasites Vectors 9:47. https://doi.org/10.1186/s13071-016-1333-8

Shaikevich, E., Patraman, I., Bogacheva, A., Rakova, V., Zelya, O., and Ganushkina, L. 2018. Invasive mosquito species Aedes albopictus and Aedes aegypti on the Black Sea coast of the Caucasus: genetics (COI, ITS2), infection with Wolbachia and Dirofilaria. Vavilov Journal of Genetics and Breeding 22(5):574-585. https://doi.org/10.18699/VJ18.397

Shaikevich, E., Bogacheva, A. S., Rakova, V., Ganushkina, L., and Ilinsky, Y. 2019. Wolbachia symbionts in mosquitoes: Intra- and intersupergroup recombinations, horizontal transmission and evolution. Molecular Phylogenetics and Evolution 134:24-34. https://doi.org/10.1016/j. ympev.2019.01.020

Shaikevich, E. V., Zakharov, I. A., and Honek, A. 2019. Ecological genetics of Adalia beetles: variability and symbiotic bacteria in European populations of the ten-spot ladybird beetle Adalia decempunctata. Ecological Genetics 17(4):37-45. https://doi.org/10.17816/ecogen17437-45

Sinkins, S.P., Braig, H. R., and O'Neill, S. L. 1995. Wolbachia superinfections and the expression of cytoplasmic incompatibility. Proceedings of the Royal Society B: Biological Sciences 261:325-330. https://doi.org/10.1098/ rspb.1995.0154

Shoemaker, D.D., Dyer, K.A., Ahrens, M., McAbee, K., and Jaenike, J. 2004. Decreased diversity but increased substitution rate in host mtDNA as a consequence of Wolbachia endosymbiont infection. Genetics 168:2049-2058. https://doi.org/10.1534/genetics.104.030890

Tikhonovich, I. A. and Provorov, N. A. 2010. Epigenetics of ecological niches. Ecological Genetics 8(4):30-38. https://doi. org/10.17816/ecogen8430-38

Turelli, M., Hoffmann, A. A., and McKechnie, S. W. 1992. Dynamics of cytoplasmic incompatibility and mtDNA variation in natural Drosophila simulans populations. Genetics 132(3):713723. https://doi.org/10.1093/genetics/132.3.713

Vinogradova, E. B., Shaikevich, E. V., and Ivanitsky, A. V. 2007. The study on the distribution of the Culex pipiens complex mosquitoes in the European part of Russia by molecular methods of their identification. Comparative Cytogenetics 1:129-138.

Werren, J.H., Zhang, W., and Guo, L. R. 1995. Evolution and phylogeny of Wolbachia: reproductive parasites of arthropods. Proceedings of the Royal Society B: Biological Sciences 261:55-63. https://doi.org/10.1098/rspb.1995.0117

Yen, J. H. and Barr, A. R. 1971. New hypothesis of the cause of cytoplasmic incompatibility in Culex pipiens. Nature 232(5313):657-658. https://doi.org/10.1038/232657a0

Zakharov, I. A. and Shaikevich, E. V. 2001. The Stockholm populations of Adalia bipunctata (L) (Coleoptera: Coccinelli- 
dae) - a case of extreme female-biased population sex ratio. Hereditas 134(3):263-266. https://doi.org/10.1111/ j.1601-5223.2001.00263.x

Zakharov, I. A. and Shaikevich, E. V. 2012. An mtDNA polymorphism in the St. Petersburg population of Adalia bipunctata and its correlation with infection by the symbiotic bacterium Spiroplasma. Russian Journal of Genetics: Applied Research 2:110-113. https://doi.org/10.1134/ S207905971202013X

Zé-Zé, L., Borges, V., Osório, H., Machado, J., Gomes, J., and Alves, M. 2020. Mitogenome diversity of Aedes
(Stegomyia) albopictus: Detection of multiple introduction events in Portugal and potential within-country dispersal. bioRxiv 2020.02.12.945741. https://doi. org/10.1101/2020.02.12.945741

Zhong, D., Lo, E., Hu, R., Metzger, M. E., Cummings, R., Bonizzoni, M., Fujioka, K. K., Sorvillo, T. E., Kluh, S., Healy, S. P., Fredregill, C., Kramer, V. L., Chen, X., and Yan, G. 2013. Genetic analysis of invasive Aedes albopictus populations in Los Angeles County, California and its potential public health impact. PLoS One 8(7):e68586. https://doi. org/10.1371/journal.pone.0068586 